Encontro Nacional de

Economia Industrial e Inovação
Indústria e Desenvolvimento Econômico:

desafios e perspectivas

18 a 20 de setembro de 2018

Uberlândia - Minas Gerais

\title{
ANÁLISE DA ADERÊNCIA DAS TAXONOMIAS INDUSTRIAIS À REALIDADE DA INDÚSTRIA DE TRANSFORMAÇÃO BRASILEIRA
}

\author{
Mabel Diz Marques* \\ José Eduardo Roselino ${ }^{\dagger}$ \\ Suelene Mascarini ${ }^{\ddagger}$
}

RESUMO: Este artigo analisa a aderência das taxonomias industriais à realidade da indústria de transformação brasileira, a partir da seleção de indicadores de esforços inovativos e tecnológicos, qualificação da mão de obra e produtividade. A abordagem fundamenta-se no arcabouço teórico neoschumpeteriano e faz o uso da análise de agrupamento. Os resultados sugerem relativa aderência aos padrões internacionais. Contudo, a atividade de refino de petróleo apresenta elevados esforços inovativos, mão de obra altamente qualificada e alta produtividade quando comparada com outras atividades no Brasil ou mundo. Neste sentido, essa atividade se diferencia da classificação da OCDE em que é apontada como média-baixa tecnologia. Em contrapartida, atividades classificadas com maior intensidade tecnológica e baseada em ciência como de instrumentos, materiais para uso médico, odontológico e de artigos ópticos estão muito aquém disso no Brasil quando avaliamos principalmente o perfil da mão de obra e a produtividade.

Palavras-Chave: Taxonomia Industrial. Intensidade Tecnológica. Análise de Cluster.

ABSTRACT: This article analyses the adherence of industrial taxonomies to the reality of the manufacture Brazilian, from the selection of indicators of innovative and technological efforts, labour qualification and productivity. This paper is based on the neoschumpeterian theoretical framework and it use of cluster analysis. The results suggest some adherence to taxonomies international, while other sectors do not. However, the petroleum refining activity presents high innovative activity, highly labour qualification and high productivity compared to other activities in Brazil or the world. In this way, this activity differs from the OECD classification in which it is referred to as medium-low technology. On the other hand, activities classified with greater technological intensity and based on science as of instruments, materials for medical, dental and optical use are far behind in Brazil when we mainly evaluate labour qualification and productivity.

Keywords: Industry Taxonomy. Technological Intensity. Cluster Analysis.

\section{ÁREA ABEIN: Área 1 - Indústria e competitividade}

1.4 Padrões de especialização produtiva e desenvolvimento

JEL: O3

\footnotetext{
*Doutoranda em Economia pela Universidade Federal da Bahia. E-mail: mabeldizmarques@gmail.com

$\dagger$ Professor do Programa de Pós-Graduação em Economia da Universidade Federal de São Carlos. E-mail:jeroselino@ufscar.br

¥ Pós-doutoranda em Economia pela Universidade Federa de São Carlos. E-mail: smascarini@gmail.com
} 


\section{Introdução}

Após o advento da revolução industrial, a dinâmica econômica passou a ser comandada por um sistema em que a inovação e as condutas inovativas tornaram instrumentos fundamentais para o desenvolvimento e crescimento econômico.

Em iniciativas voltadas à identificação dos padrões setoriais de inovação tecnológica foram construídas taxonomias industriais que buscam agregar características conforme determinados critérios de análise. A taxonomia setorial proposta por Pavitt (1984) e a classificação tecnológica da Organização para a Cooperação e Desenvolvimento Econômico - OCDE (2011) são as mais empregadas e difundidas por formuladores de políticas e por pesquisadores da área de economia da inovação, nas quais possibilitam comparações entre países e atividades econômicas segundo Cavalcante (2014).

A taxonomia Pavitt (1984) descreve os padrões setoriais das mudanças técnicas a partir das semelhanças das inovações realizadas pelas empresas e setores no Reino Unido. A taxonomia busca identificar setores tipicamente associados às três categorias, sendo elas: i) Baseadas em Ciência, ii) Produção Intensiva e iii) Dominados pelo Fornecedor conforme suas trajetórias tecnológicas setoriais.

Já a classificação tecnológica da OCDE (2011) registrada por Hatzichronoglou (1997), corresponde uma agregação dos setores da indústria de transformação por nível tecnológico. As atividades são categorizadas e baseadas no processo de produção em quartis denominados em alta, média-alta, médiabaixa e baixa intensidade tecnológica em função do valor da relação dos gastos em Pesquisa e Desenvolvimento (P\&D) sobre a receita líquida de vendas setorial.

Apesar do marco e relevância da taxonomia Pavitt (1984) e classificação OCDE (2011) para o tema, há um grande desafio à ciência em classificar setores diante dos fenômenos evolucionários, e especificidades regionais e locais. De Marchi, Napolitano e Taccini (1996) testaram um modelo para as trajetórias tecnológicas da indústria manufatureira da Itália baseada na taxonomia de Pavitt (1984), os resultados não demonstraram inconsistência com a taxonomia Pavitt, muito embora ressalte o desafio à identificação de um padrão industrial de inovação tecnológica.

Adicionalmente, como a taxonomia Pavitt (1984) e classificação OCDE (2011) não prestaram ao exame dos países em desenvolvimento - como é o caso brasileiro - os indicadores não são qualitativamente suficientes para compreensão do padrão setorial de inovação tecnológica nos países em desenvolvimento. Estudos prévios, como Furtado e Quadros (2005), Guidolin e Martinelli (2008) e Silva e Suzigan (2014) identificaram que existem fatores críticos que explicam diferenças em relação aos padrões setoriais existentes nos países desenvolvidos.

É essa lacuna que o presente artigo pretende preencher ao investigar, a partir da análise conjunta de três diferentes dimensões (atividade inovativa, qualificação da mão de obra e produtividade), a adequação da taxonomia de Pavitt (1984) e da classificação por intensidade tecnológica da OCDE (2011) para a indústria de transformação brasileira. Assim, objetiva-se responder ao seguinte questionamento: as principais taxonomias consagradas refletem as reais peculiaridades da estrutura produtiva das economias em desenvolvimento, e particularmente ao caso brasileiro? Uma vez que essas taxonomias não foram construídas com base no exame dos países em desenvolvimento configura-se um grande desafio comparar padrões setoriais de estruturas socioeconômicas distintas. Além disso, em alguns casos a estrutura produtiva pode refletir uma configuração em que a produção doméstica se dá com base em esforços tecnológicos externos. Ou seja, a estrutura produtiva pode ter nessas situações características semelhantes à de outros países em termos de configurações setoriais, mas com menor esforço tecnológico que eles (FURTADO; CARVALHO, 2005; SILVA; SUZIGAN, 2014; GONÇALVES; YONAMINI, 2013). Assim, faz-se necessária uma análise da adequação das taxonomias à realidade brasileira sob a hipótese de que as categorias setoriais (atividades econômicas) das principais taxonomias amplamente difundidas internacionalmente - Pavitt (1984) e OCDE (2011) -, possuem características e comportamentos distintos de esforços tecnológicos da indústria de transformação brasileira, principalmente, em setores com menor intensidade tecnológica e produção intensiva em escala, em virtude do diferencial das trajetórias tecnológicas entre os países desenvolvidos e em desenvolvimento. 
Os resultados dessa análise apontam que algumas atividades econômicas da indústria de transformação no Brasil apresentam um padrão bastante peculiar, distanciando assim das principais classificações já consagradas na literatura. Destaque deve ser dado principalmente à atividade de refino de petróleo na qual pode ser caracterizada por uma atividade de média-alta atividade inovativa e alta qualificação da mão de obra e produtividade. Esse resultado demonstra não somente a importância dessa atividade na indústria brasileira, mas também seu diferencial comparado à classificação da OCDE que a coloca como uma atividade de média-baixa intensidade tecnológica.

Além dessa introdução, este artigo está organizado em três seções e consideração final. A primeira seção busca recuperar e sintetizar a construção das taxonomias industriais empregadas neste artigo (Pavitt (1984) e OCDE (2011)), a partir das características conceituais, metodológicas e operacionais. A segunda seção descreve os procedimentos metodológicos, por meio da análise de agrupamento (Clusters Analysis) técnica estatística multivariada. A terceira seção discute os resultados gerados na seção anterior. Por fim, a conclusão apresenta algumas reflexões finais, bem como limitações e sugestões para o aprimoramento de pesquisas sobre aderência dos padrões setoriais de inovação tecnológica na indústria de transformação brasileira.

\section{Taxonomias e classificações setoriais: Uma revisão teórico-metodológico}

Tradicionalmente as taxonomias tiveram por função classificar as espécies nas ciências biológicas, surgindo como ciência das leis das classificações de formas vivas. Por definição, taxonomias são classificações gerais que procuram reunir grupos semelhantes de acordo com todos os seus atributos comuns, sendo "obtidas de objetos reais baseadas em uma ou mais características, tipificada pela aplicação de análise de agrupamento ou outros procedimentos de agregação." (HAIR ET AL., 2009, p.429).

No estudo da economia da inovação, surgiram diversas propostas de taxonomias baseadas em indicadores de esforços tecnológicos, com intuito de explicar os diferentes padrões setoriais. Fundamentadas, sobretudo, pela teoria evolucionista ou comumente denominada Neoschumpeteriana, os estudos desta linha teórica procuram identificar as diferenças intersetoriais na forma de buscar, introduzir, utilizar e difundir inovações na dinâmica capitalista, ou seja, o ambiente tecnológico em que as empresas operam (DOSI, 1982; 1988; NELSON E WINTER, 1982; PAVITT, 1984; ROBSON; TOWNSEND; PAVITT, 1988).

Pavitt (1984) a partir de um conjunto de indicadores relacionados aos esforços e resultados inovativos, tamanho e produtividade das empresas, de uma amostra com aproximadamente 2000 inovações significativas entre as décadas de 1940 e 1970, identificadas por especialistas setoriais independente das firmas no Reino Unido descreve e explica os padrões setoriais de mudanças técnicas, a partir das semelhanças e diferenças entre os setores, ao classificar as firmas conforme as competências e trajetórias tecnológicas, considerando que mudanças tecnológicas seriam processos dinâmicos e cumulativos propõe três categorias dos setores industriais, sendo elas: i) dominada pelo fornecedor; ii) produção intensiva e iii) baseadas em ciência. (GUIDOLIN; MARTINELLI, 2008; CARLIN; CHEVARRIA, 2012).

A categoria setorial dominada pelo fornecedor caracteriza-se por baixos gastos com $\mathrm{P} \& \mathrm{D}$, os aprimoramentos são em sua maioria incrementais, as firmas são relativamente pequenas, a cumulatividade e apropriabilidade tecnológicas são restritas. Segundo Arend (2009), as inovações das fïmas desta categoria são predominantemente inovações de processo, incorporadas em equipamentos de capital e insumos intermediários originados em firmas de outros setores, cuja atividade principal é fornecer bens de produção a setores tradicionais. Os setores típicos são agricultura, têxtil, vestuário, couro, imprensa, construção civil, produção familiar informal, serviços profissionais, financeiros, comerciais, produtos de madeira e produtos metálicos simples.

As firmas categorizadas com produção intensiva dividem-se em: a) fornecedores especializados ou difusores do progresso técnico. As inovações desta categoria têm características a inserção em vários outros setores como insumos de capital. Exemplos setoriais são indústrias de instrumentos mecânicos e engenharia. Já as firmas, são geralmente pequenas e localizadas próxima aos usuários, os conhecimentos são especializados e tácitos, além de existir elevada oportunidade e apropriabilidade tecnológica; b) 
intensivos em escala. As inovações relacionadas a esta categoria estão associadas às inovações de processos e produtos, embora, a incidência das inovações incrementais (de processo) sejam mais propícias a ocorrer do que inovações radicais (de produto). As atividades de produção envolvem sistemas complexos dominados com maestria e, frequentemente, industrialização de produtos complexos. Exemplos são os setores de equipamentos de transporte, bens de consumo duráveis, (eletroeletrônicos), metalurgia, alimentos, vidro, cimento, construção naval, fabricação e montagem de máquinas, que em geral possuem grandes plantas industriais com elevada integração vertical.

Já nas firmas classificadas como baseadas em ciência, as inovações são diretamente relacionadas com os novos paradigmas tecnológicos, viabilizados pelo avanço tecnológico. Os setores típicos são a indústria eletrônica, indústria química (orgânica), farmacêutica e bioengenharia. As oportunidades tecnológicas são muito elevadas, ocorrem altos gastos em P\&D (laboratórios formalizados), uma parcela elevada de seus produtos é insumo para setores de bens de capital e intermediários e as firmas tendem a ser grandes com exceções de inovadores pequenos, altamente especializados (AREND, 2009).

Além da Taxonomia proposta por Pavitt (1984) a classificação da OCDE (2011) é também amplamente difundida nos estudos da economia da inovação. A classificação por intensidade tecnológica da OCDE (2011) é uma classificação setorial baseada nos processos de produção. Os setores industriais são agregados de acordo com a intensidade tecnológica em quatro grupos: alta, média-alta, média-baixa e baixa (HATZICHRONOGLOU, 1997).

Inicialmente, o indicador empregado foi obtido através da divisão dos gastos com $\mathrm{P} \& \mathrm{D}$ pelo valor adicionado, gastos em bens intermediários e capitais ou produção, onde "os trabalhos exploravam indicadores de fluxos tecnológicos intersetoriais construídos a partir da matriz insumo-produto" (FURTADO E CARVALHO, 2005, p.72). Após diversas revisões desde a sua primeira publicação, "o índice do indicador restringiu a classificação por intensidade tecnológica pelos gastos em P\&D" (CAVALCANTE, 2014, p.5).

A classificação tecnológica OCDE (2011) possibilita comparações internacionais dos esforços tecnológicos dos setores industriais, uma vez que estes não estão distribuídos uniformemente entre os países. As classificações setoriais baseadas nos processos de produção tal como a OCDE (2011) envolvem:

“i) International Standard Industrial Classification of All Economic Activities (ISIC) da Divisão Estatística das Nações Unidas; ii) North American Industry Classification System (NAICS); iii) Statistical Classification of Economic Activities in the European Community (NACE); iv) Classificação Nacional de Atividades Econômicas (CNAE), usada no Brasil; v) outras classificações setoriais baseadas nos processos de produção e usadas em diferentes países. Essas classificações são permanentemente revisadas, havendo, portanto, diferentes versões de cada uma delas. A correspondência entre os setores definidos nessas classificações é amplamente documentada. Em suas versões mais recentes, a ISIC, a NAICS, a NACE e a CNAE mantêm uma evidente correspondência entre si. Todas essas classificações são formadas, assim, por seções (21, no caso na CNAE revisão 2.1), divisões (87), grupos (285) e classes (673). Por serem identificadas por dois dígitos numéricos, as divisões são usualmente referidas como "CNAE dois dígitos". Nesse nível de agregação, as indústrias extrativas (seção "B") são formadas por cinco divisões (de 05 a 09) e as indústrias de transformação (seção "C") são formadas por 24 divisões (de 10 a 33). Essa estrutura é compatível com a revisão 4.0 da ISIC e com as versões mais recentes da NAICS e da NACE (cujas estruturas refletem a ISIC).” (CAVALCANTE, 2014, p. 3).

A primeira definição e classificação de intensidade tecnológica proposta por Hatzichronoglou (1997) foi em ISIC Rev. 2, e foi amplamente difundida. A metodologia fundamenta-se tanto na intensidade direta de Pesquisa e Desenvolvimento (P\&D), quanto na incorporação dos investimentos nos bens intermediários. Em 2003, a classificação foi revisitada e a definição foi atualizada para ISIC Rev. 3 utilizando dados de 1991 a 1999. O indicador proposto para ponto de corte foi estabelecido, através da relação dos gastos diretos de $\mathrm{P} \& \mathrm{D}$ pelo valor agregado e valor bruto da produção. Vale destacar que, ao adicionar a intensidade indireta de Investigação e Desenvolvimento (I\&D) ocorreram modificações do 
ranking dos setores industriais. Porém, não modificou a composição dos grupos ao utilizar apenas intensidade direta de I\&D. A revisão mais recente da classificação setorial refere-se a 2011.

É importante ressaltar, que a classificação da OCDE não pressupõe, a priori, nenhum tipo de homogeneidade, em cada grupo, dos padrões setoriais de mudança técnica. Com isso, é possível que determinados setores que compõem uma classe reajam de forma bastante distinta aos estímulos colocados por ações específicas de políticas. Dessa forma, não há porque supor que os estímulos eficazes para um determinado setor de alta tecnologia, por exemplo, sejam para outros setores que pertençam ao mesmo grupo (CAVALCANTE, 2014).

No âmbito brasileiro algumas pesquisas têm desprendido esforços para contribuir para avaliação da aderência das taxonomias mencionadas, além de propor novas taxonomias. De modo geral os resultados desses estudos têm demostrado que apenas parte das taxonomias é convergente com a realidade brasileira (QUADROS, 2005; GUIDOLIN E MARTINELLI, 2008; CAMPOS E RUIZ, 2009; CASTRO 2010; GONÇALVES E YONAMINI, 2013; SILVA E SUZIGAN, 2014).

Para Campos e Ruiz (2009) e Castro (2010) não foi possível identificar atividades econômicas com padrões setoriais semelhantes aos fornecedores especializados a partir da taxonomia Pavitt (1984). Já Silva e Suzigan (2014) apontam que setores da indústria de fumo, couro, celulose, metalurgia básica, alimentos e bebidas apresentam baixa aderência com a taxonomia. Em tese, essa divergência ocorre pela heterogeneidade intrasetorial e peculiaridades econômicas associadas ao Brasil.

No que tange à classificação tecnológica, Furtado e Quadros (2005) sugere uma classificação alternativa para indústria de transformação que eventualmente seja ponto de partida para uma reflexão mais sistemática sobre as características estruturais e diferenciadas das trajetórias tecnológicas de países em desenvolvimento, uma vez que os autores concluem que os esforços tecnológicos empreendidos em setores de alta intensidade tecnológica no Brasil em termos proporcionais são relativamente inferiores ao realizado nos países desenvolvidos. Adicionalmente, Guidolin e Martinelli (2008) e Gonçalves e Yonamini (2013) apontam que a indústria brasileira possui regimes tecnológicos diferentes dos definidos para os países desenvolvidos, reforçando a importância de estudos sobre as características de mudança técnica nos países em desenvolvimento.

Neste contexto, observa-se uma multiplicidade de classificações e refinamentos que buscam investigar o padrão dos esforços inovativos da indústria de transformação brasileira, por meio da combinação de indicadores cienciométricos e de estrutura de mercado certamente pela emergência de uma taxonomia nacional, pois os critérios para criar os agrupamentos, ou seja, para demonstrar homogeneidade e heterogeneidade entre as atividades econômicas são inúmeras e as trajetórias tecnológicas dos países distintas. Além disso, é importante ressaltar o desafio das ciências em classificar e adotar padrões setoriais excessivamente "rígidos" diante das especificidades, dinâmica industrial e fenômenos evolucionários. Desta forma, faz-se necessário a construção de indicadores que reproduzam novos olhares e indiquem ao longo do processo, as possibilidades de mensuração dos esforços inovativos e padrões tecnológicos que vão além de indicadores tradicionais de esforços inovativos, incorporando características que dizem respeito à natureza da força de trabalho e conteúdo do trabalho dada a relevância do conhecimento tácito para o processo de geração e difusão de conhecimento e de alguma forma estão relacionadas com atividades de ciência, tecnologia e inovação $(\mathrm{C}, \mathrm{T} \& \mathrm{I})$.

\section{Dados e Metodologia}

Para analisar a adequação da taxonomia Pavitt (1984) e da classificação tecnológica proposta pela OCDE (2011) para as atividades econômicas da indústria de transformação brasileira foi selecionado um conjunto de dados que estão identificados em 28 atividades econômicas ${ }^{4}$ da indústria de transformação (24 setores desagregados a dois níveis (divisão) e quatro setores a três níveis (grupo) da classificação nacional de atividades econômicas - CNAE 2.0). Essas atividades selecionadas são classificadas neste trabalho de acordo com as taxonomias analisadas conforme as características de intensidade tecnológica (alta, média-

\footnotetext{
${ }^{4}$ A desagregação das atividades econômicas está descrita no Anexo A
} 
alta, média-baixa e baixa tecnologia) e categorias setoriais (Baseada em ciência, Produção intensiva em escala, Fornecedor especializado e Dominado pelo fornecedor).

A base de dados utilizada nesse trabalho foi construída a partir de um conjunto de dados secundários provenientes das seguintes fontes de informação: Pesquisa de Inovação (PINTEC) do Instituto Brasileiro de Geografia e Estatística (IBGE); Pesquisa Industrial Anual (PIA-Empresa) do IBGE e Relação Anual de Informações Sociais (RAIS) do Ministério do Trabalho e Emprego (MTE). O estudo está limitado ao ano 2014 principalmente devido aos dados da PINTEC, último ano da pesquisa. Na tabela 1 tem-se cada indicador utilizado, medida e fonte de informação.

Tabela 1 - Indicador, variável e fonte de informações

\begin{tabular}{|c|c|c|c|}
\hline Dimensão & Indicador & Medida & Fonte \\
\hline \multirow{4}{*}{$\begin{array}{l}\text { Atividade } \\
\text { Inovativa }\end{array}$} & TaxInov & $\begin{array}{l}\text { \% número de empresas que realizaram inovação de produto e/ou } \\
\text { processo no setor }\end{array}$ & \multirow{4}{*}{ PINTEC } \\
\hline & $P D$ & $\begin{array}{l}\text { Dispêndio das empresas inovadoras nas atividades inovativas em } \\
\text { atividades internas de } \mathrm{P} \& \mathrm{D} / \text { receita líquida de vendas no setor }\end{array}$ & \\
\hline & Soft & $\begin{array}{l}\text { \% de dispêndio das empresas inovadoras em aquisição de } \\
\text { software/ receita líquida de vendas no setor }\end{array}$ & \\
\hline & $M a q$ & $\begin{array}{l}\text { \% de dispêndio das empresas inovadoras em aquisição de } \\
\text { máquinas e equipamentos/ receita líquida de vendas no setor }\end{array}$ & \\
\hline \multirow{3}{*}{$\begin{array}{l}\text { Perfil da Mão } \\
\text { de Obra }\end{array}$} & Escol & \% empregada com ensino superior completo & \multirow{3}{*}{ RAIS } \\
\hline & $C T$ & $\%$ de pesquisadores e engenheiros empregados & \\
\hline & Tec & $\%$ de empregados nas ocupações técnicas & \\
\hline \multirow[b]{2}{*}{ Produtividade } & $R L V$ & Receita líquida de vendas/ PO (pessoal ocupado em 31.12.2014) & \multirow[b]{2}{*}{$\begin{array}{l}\text { PIA- } \\
\text { Empresa }\end{array}$} \\
\hline & $V T I$ & $\begin{array}{l}\text { Valor da transformação Industrial/ PO (pessoal ocupado em } \\
31.12 .2014 \text { ) }\end{array}$ & \\
\hline
\end{tabular}

Fonte: Elaboração própria (2018)

Como é possível observar na tabela 1 os indicadores foram divididos em três dimensões: atividade inovativa, perfil da mão de obra e produtividade. A primeira dimensão, atividade inovativa, é composta por quatro indicadores com dados provenientes da PINTEC. TaxInov representa a taxa de inovação em cada setor é medida pela participação das empresas inovativas em cada setor. $\boldsymbol{P D}$ é o indicador de esforço inovativo em $\mathrm{P} \& \mathrm{D}$ na atividade, medido pelos dispêndios em atividades internas de $\mathrm{P} \& \mathrm{D}$ em relação à receita líquida de vendas. Soft representa os esforços com software e é mensurado pelos dispêndios em aquisição de software na atividade em relação à indústria de transformação. Já Maq é o indicador de esforço em adquirir máquinas e equipamentos, medido pelo gasto na aquisição de máquinas e equipamentos na atividade em relação à indústria de transformação. Tem como hipótese que elevado dispêndio empreendido com a aquisição Soft e $\boldsymbol{M a q}$ derivem em baixos esforços de produção do conhecimento interno via P\&D, pois para acompanhar as mudanças técnicas esses setores associam-se à incorporação de inovação tecnológica externa. Neste sentido, maiores valores da taxa de inovação e P\&D e menores valores dos esforços em adquirir máquinas e softwares representam mais alta atividade inovativa.

A segunda dimensão é o perfil da mão de obra, para tal utilizam-se três indicadores: Escol representa a qualificação da mão de obra e é mensurado pela participação dos empregados com ensino superior na atividade. $\boldsymbol{C T}$ composto por trabalhadores que transitam de modo direto em atividades de ciência, tecnologia e inovação mensurado exclusivamente pela participação de pesquisadores e engenheiros nas atividades. Tec representa a participação de empregados nas ocupações técnicas especificas com elevado conteúdo de capacidade tácita incorporado nas rotinas operacionais. Para os indicadores $\boldsymbol{C T}$ e $\boldsymbol{T e c}$ são selecionadas 51 famílias ocupacionais potencialmente vinculadas às atividades e processos de geração e 
difusão de conhecimento técnicos e científicos na CBO sendo estas hierarquizadas por critérios ad hoc e proveniente da base de dados secundários da RAIS/MTE ${ }^{5}$. Indicadores dessa natureza potencializa o processo de identificação dos esforços inovativos e reduzem a limitação de classificações que consideram os gastos em P\&D formal como única proxy de esforços inovativos, tais como a OCDE (2011).

Por fim, a terceira dimensão analisada nesse trabalho é a produtividade, a partir de dois indicadores com dados oriundos da PIA-Empresa. $\boldsymbol{R} \boldsymbol{V} \boldsymbol{L}$ é a receita líquida de vendas no setor (atividade) em relação ao pessoal ocupado e $\boldsymbol{V T I}$ é medido pelo valor da transformação industrial em relação ao pessoal ocupado.

Este trabalho faz uso da análise de cluster. A análise de cluster é uma técnica multivariada de classificação que visa agrupar dados por meio das similaridades ou dissimilaridades entre eles. Assim, agrupa um conjunto de dados heterogêneos em grupos mais homogênea seguindo um critério fixado. Essa técnica tem sido bastante utilizada na literatura e envolve uma série de distintos métodos e algoritmos para agrupar objetos/indivíduos do mesmo tipo nas respectivas categorias (JOHNSON, 1967; GONÇALVES E SIMÕES, 2005; FIELDING, 2007; CARVALHO, 2009; LINDEN, 2009; CASTRO, 2010; SILVA, 2013). Portanto, a análise de cluster é uma ferramenta de análise que busca à triagem de diferentes objetos/indivíduos em grupos, de modo que o grau de associação entre dois objetos é máximo, se eles pertencem ao mesmo grupo, e mínimo em caso contrário. Assim, a análise de cluster não é uma análise que permite explicar porque as estruturas de dados existem, mas sim descobrir como elas ocorrem.

Como apontado anteriormente, os métodos e algoritmos da análise de cluster são variados e dependem de um conjunto de hipóteses e análises para serem definidos.

Este trabalho utiliza-se de um processo hierárquico a partir das dissemelhanças entre dos casos $(i \mathrm{e}$ $j$ ). A medida de dissimilaridade escolhida foi a distância euclidiana ao quadrado ${ }^{6}$. Além disso, como método de agrupamento adotou-se o Ward, esse também denominado método de variância é um procedimento de agrupamento que se inicia com todos os objetos/indivíduos em um único grupo e então vai formando os subgrupos. Esse método tende a resultar grupos de tamanhos aproximados, devido a minimização da variação interna. O método de Ward permite a representação dos resultados na forma gráfica conhecida por dendograma que será apresentada nos resultados. Ressalta-se que outras métodos e algoritmos foram testados nesse trabalho e a apresentação desse se faz pois foi o que mais se adequa do estudo em questão. Deve-se apontar que ainda que os indicadores tenham sido separados em três dimensões nesse trabalho, a análise de cluster foi aplicada a todos os indicadores conjuntamente.

Antes de apresentar os resultados, aponta-se que ao aplicar a técnica hierárquica de agrupamento aglomerativo por meio da distância euclidiana ao quadrado e utilizar o método Ward, o coeficiente de correlação cofenética (ccc) ${ }^{7}$ encontrado foi de 0,86 que valida o elevado grau de ajuste a matriz de dissimilaridade com o dendograma.

Realizado os agrupamentos, os indicadores serão apresentados em cada grupo e individualmente em cada atividade para a análise em questão.

\section{Resultados e discussão}

As 28 atividades econômicas foram separadas em quatro grupos, sendo possível identificar o ponto de corte na distância 24, valor que corresponde a $16 \%$ da distância máxima observada nos níveis de fusão (figura 1). Dado a utilização do método aglomerativo os casos mais similares são fundidos mais próximos da base e os mais dissimilares ao longo do dendograma. Já na tabela 2 é apresentada cada cluster e suas atividades com seus respectivos indicadores.

\footnotetext{
${ }^{5}$ As famílias ocupacionais selecionadas são apresentadas no Anexo B

${ }^{6}$ A distância euclidiana ao quadrado é obtido pela equação $d_{i j}=\operatorname{dist}\left(\mathrm{E}_{\mathrm{i}}, \mathrm{E}_{\mathrm{j}}\right)=\sum_{\mathrm{k}=1}^{\mathrm{p}}\left(\mathrm{x}_{\mathrm{ik}}-\mathrm{x}_{\mathrm{jk}}\right)^{2}$. Onde: $d_{i j}^{2}=$ quadrado da distância euclidiana entre $i$ e $j ; \mathrm{x}_{\mathrm{ik}} \mathrm{e}\left(\mathrm{x}_{\mathrm{jk}}\right)=$ atributo do $i$-ésimo ( $j$-ésimo) componente na $k$-ésima variável; $p=$ números de variáveis. A principal vantagem dessa medida está associada a elevada adequabilidade aos diversos algoritmos dos métodos hierárquicos. A similaridade entre os indivíduos será maior quando o valor da distância for mais próximo de zero.

${ }^{7} \mathrm{O}$ cálculo do coeficiente ccc resulta em um valor entre 0 (zero) e 1 (um). Assim, o menor grau de distorção é refletido pelo maior coeficiente (ccc), sendo este capaz de evidenciar corretamente a melhor estrutura dos dados e formação do dendograma. De acordo com Rohlf (1970), ccc superior a 0,7 indica adequabilidade do grau de ajuste do método de agrupamento para resumir as informações do conjunto de dados.
} 
Figura 1 - Dendograma obtido com base na distância euclidiana ao quadrado e método Ward para agrupamento de 28 atividades econômicas e 9 indicadores selecionados

Cluster Dendrogram
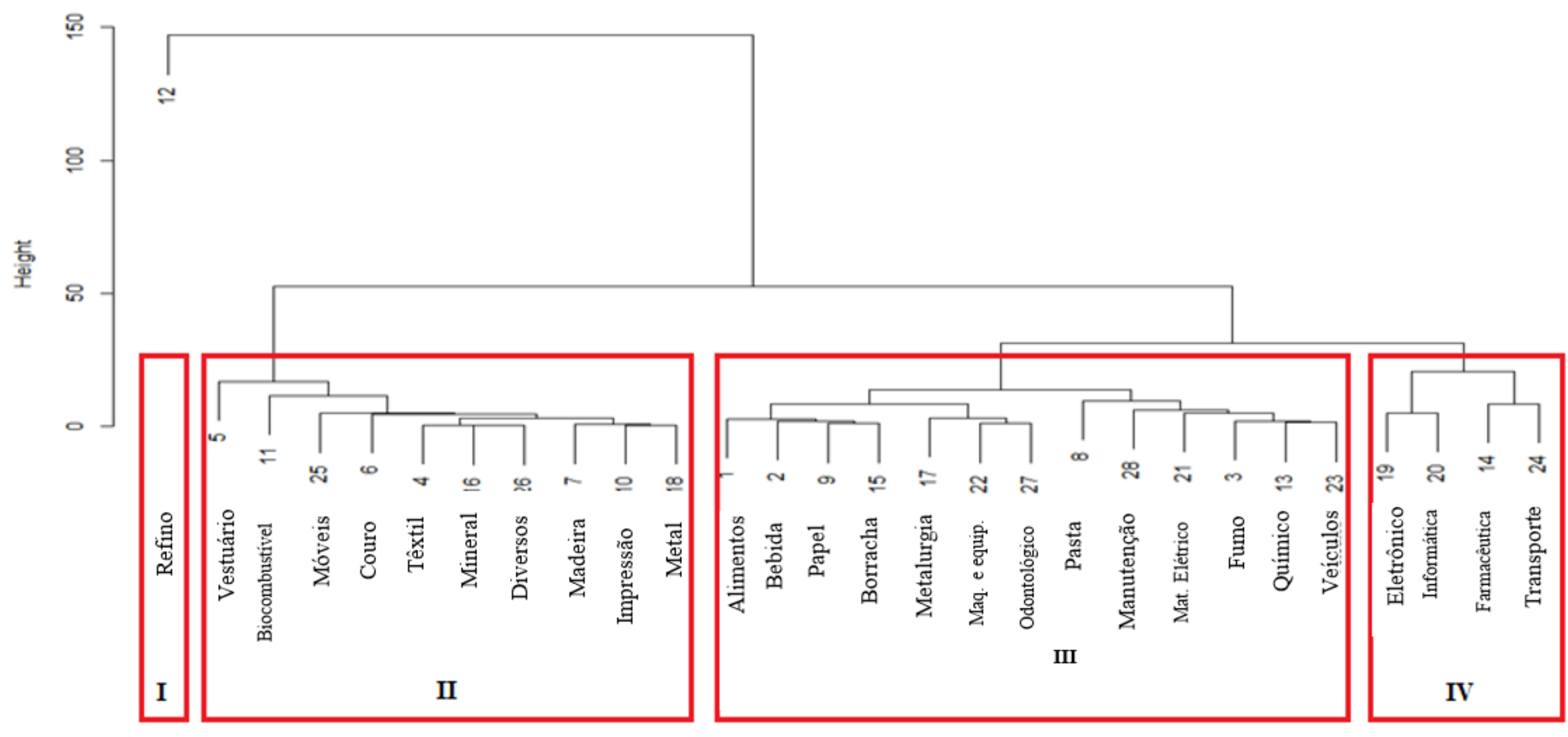

d1

hclust (", "ward.D2")

Fonte: Elaboração própria (2018) com base na saída do RStudio 
Tabela 2 - Resultados dos indicadores

\begin{tabular}{|c|c|c|c|c|c|c|c|c|c|c|c|c|}
\hline \multirow{3}{*}{ ID } & \multirow{3}{*}{ Cluster/ Classificação Nacional de Atividade Econômica (CNAE) } & \multirow{2}{*}{\multicolumn{2}{|c|}{ Taxonomia }} & \multicolumn{9}{|c|}{ DIMENSÃO } \\
\hline & & & & \multicolumn{4}{|c|}{ Atividade inovativa } & \multicolumn{3}{|c|}{$\begin{array}{c}\text { Perfil da mão de } \\
\text { obra }\end{array}$} & \multicolumn{2}{|c|}{ Produtividade } \\
\hline & & $\begin{array}{c}\text { Pavitt } \\
(1984)^{*}\end{array}$ & $\begin{array}{c}\text { OCDE } \\
(2011)^{* *}\end{array}$ & $\begin{array}{c}\text { TaxInov } \\
(\%)\end{array}$ & $P D$ & $\begin{array}{l}\text { Soft } \\
(\%)\end{array}$ & $\begin{array}{c}\text { Maq } \\
(\%)\end{array}$ & $\begin{array}{c}\text { Escol } \\
(\%)\end{array}$ & $\begin{array}{r}C T \\
(\%)\end{array}$ & $\begin{array}{l}\text { Tec } \\
(\%)\end{array}$ & $R L V$ & $V T I$ \\
\hline \multicolumn{2}{|c|}{ C- Indústria de Transformação } & - & - & 36,30 & 0,7 & 0,05 & 0,86 & 9,5 & 1,0 & 5,4 & 340,3 & 137,7 \\
\hline- & Cluster I & - & - & 52,36 & $\mathbf{0 , 9}$ & $\mathbf{0 , 0 1}$ & $\mathbf{0 , 1 1}$ & 66,3 & $\mathbf{1 8 , 5}$ & 11,7 & $4.367,8$ & 2.089,4 \\
\hline 12 & Refino de petróleo & PIE & MB & 52,36 & 0,9 & 0,01 & 0,11 & 66,3 & 18,5 & 11,7 & $4.367,8$ & $2.089,4$ \\
\hline- & Cluster II & - & - & 33,28 & $\mathbf{0 , 3}$ & $\mathbf{0 , 1 1}$ & $\mathbf{1 , 5 6}$ & 5,2 & $\mathbf{0 , 3}$ & 2,8 & 166,3 & $\mathbf{7 9 , 0}$ \\
\hline 4 & Fabricação de produtos têxteis & DF & B & 32,02 & 0,1 & 0,06 & 1,30 & 4,5 & 0,1 & 3,5 & 149,4 & 59,9 \\
\hline 5 & Confecção de artigos do vestuário e acessórios & DF & B & 29,04 & 0,2 & 0,27 & 0,75 & 3,4 & 0,0 & 1,6 & 82,7 & 45,3 \\
\hline 6 & Preparação de couros e fabricação de artefatos de couro, artigos de viagem e calçados & DF & B & 28,76 & 0,7 & 0,06 & 0,56 & 4,1 & 0,1 & 1,9 & 104,8 & 50,2 \\
\hline 7 & Fabricação de produtos de madeira & DF & B & 25,30 & 0,2 & 0,06 & 1,87 & 3,2 & 0,2 & 1,4 & 149,1 & 67,4 \\
\hline 10 & Impressão e reprodução de gravações & DF & $\mathrm{B}$ & 27,63 & 0,4 & 0,12 & 1,76 & 9,8 & 0,1 & 2,9 & 221,3 & 120,2 \\
\hline 11 & Fabricação de coque e biocombustíveis (álcool e outros). Exceto ID 12 & PIE & MB & 38,20 & 0,1 & 0,03 & 2,95 & 5,8 & 0,6 & 4,2 & 236,5 & 104,0 \\
\hline 16 & Fabricação de produtos de minerais não metálicos & PIE & MB & 38,51 & 0,3 & 0,07 & 1,55 & 5,1 & 0,5 & 3,1 & 225,4 & 107,0 \\
\hline 18 & Fabricação de produtos de metal. & PIE & MB & 29,44 & 0,3 & 0,09 & 2,07 & 6 & 0,6 & 4,8 & 210,2 & 91,7 \\
\hline 25 & Fabricação de móveis & DF & $\mathrm{B}$ & 44,01 & 0,5 & 0,21 & 1,51 & 4,0 & 0,1 & 1,6 & 143,7 & 63,7 \\
\hline 26 & Fabricação de produtos diversos. Exceto ID 27 & FE & $\mathrm{B}$ & 39,92 & 0,2 & 0,09 & 1,23 & 5,9 & 0,2 & 3,1 & 139,5 & 80,5 \\
\hline- & Cluster III & - & - & 36,30 & $\mathbf{0 , 7}$ & $\mathbf{0 , 0 5}$ & $\mathbf{0 , 8 6}$ & $\mathbf{1 3 , 8}$ & $\mathbf{1 , 5}$ & 8,7 & 465,0 & 209,9 \\
\hline 1 & Fabricação de produtos alimentícios & PIE & B & 44,55 & 0,1 & 0,03 & 0,80 & 8,1 & 0,3 & 3,3 & 306,3 & 114,1 \\
\hline 2 & Fabricação de bebidas & PIE & B & 43,73 & 0,1 & 0,01 & 1,82 & 13,6 & 0,5 & 7,1 & 451,6 & 237,2 \\
\hline 3 & Fabricação de produtos do fumo & PIE & B & 34,99 & 0,3 & 0,02 & 0,23 & 17,6 & 1,6 & 10,4 & 739,8 & 413,9 \\
\hline 8 & Fabricação de celulose e outras pastas & DF & B & 28,67 & 0,3 & 0,01 & 0,22 & 10,0 & 2,5 & 15,1 & 964,5 & 569,1 \\
\hline 9 & Fabricação de papel, embalagens e artefatos de papel - Exceto ID 8 & DF & B & 30,35 & 0,4 & 0,04 & 1,38 & 24,1 & 0,7 & 5,7 & 345,6 & 156,0 \\
\hline 13 & Fabricação de produtos químicos & $\mathrm{BC}$ & MA & 49,61 & 0,7 & 0,04 & 0,49 & 20,3 & 2,0 & 8,7 & 842,9 & 272,1 \\
\hline 15 & Fabricação de artigos de borracha e plástico & PIE & MB & 40,92 & 0,5 & 0,04 & 1,11 & 7,0 & 0,6 & 7,5 & 242,0 & 98,1 \\
\hline 17 & Metalurgia & PIE & MB & 37,64 & 0,3 & 0,06 & 1,02 & 12,4 & 2,2 & 11,7 & 716,9 & 247,0 \\
\hline 21 & Fabricação de máquinas, aparelhos e materiais elétricos & FE & MA & 47,09 & 1,8 & 0,03 & 0,64 & 12,8 & 2,2 & 8,1 & 284,8 & 114,7 \\
\hline 22 & Fabricação de máquinas e equipamentos & PIE & MA & 40,34 & 0,8 & 0,10 & 0,87 & 14,5 & 2,0 & 8,9 & 320,5 & 133,7 \\
\hline 23 & Fabricação de veículos automotores, reboques e carrocerias & PIE & MA & 39,08 & 1,1 & 0,03 & 0,68 & 18,7 & 2,8 & 9,0 & 503,7 & 173,6 \\
\hline 27 & Fabricação de instrumentos e mat. para uso médico e odontológico e de artigos ópticos & $\mathrm{BC}$ & A & 50,47 & 1,0 & 0,12 & 1,23 & 11,8 & 0,9 & 7,8 & 170,6 & 108,6 \\
\hline 28 & Manutenção, reparação e instalação de máquinas e equipamentos & FE & MA & 22,92 & 0,8 & 0,04 & 0,69 & 8,0 & 1,3 & 10,0 & 156,1 & 90,8 \\
\hline- & Cluster IV & - & - & 58,03 & 1,9 & $\mathbf{0 , 0 7}$ & $\mathbf{0 , 7 5}$ & 21,3 & 2,8 & 12,3 & 463,2 & 166,7 \\
\hline 14 & Fabricação de produtos farmoquímicos e farmacêuticos & $\mathrm{BC}$ & A & 52,18 & 2,2 & 0,07 & 0,64 & 37,2 & 1,2 & 10,5 & 368,8 & 215,9 \\
\hline 19 & Fabricação de equipamentos de inform., produtos eletrônicos e ópticos. Exceto ID 20 & $\mathrm{BC}$ & A & 67,23 & 2,0 & 0,06 & 0,48 & 17,4 & 3,4 & 13,7 & 523,3 & 179,9 \\
\hline 20 & Fabricação de equipamentos de informática e periféricos & $\mathrm{BC}$ & A & 74,75 & 1,1 & 0,02 & 0,20 & 15,8 & 2,0 & 17,1 & 584,8 & 117,0 \\
\hline 24 & Fabricação de outros equipamentos de transporte, exceto veículos automotores & FE & MA & 37,97 & 2,3 & 0,11 & 1,66 & 14,7 & 4,7 & 8,0 & 375,7 & 153,8 \\
\hline
\end{tabular}

Fonte: Elaboração própria (2018) com base no IBGE (2016a; 2016b) RAIS (2017)

$* \mathrm{BC}=$ Baseado em Ciência PIE = Produção intensiva em escala; FE = Fornecedores especializados; DF = Dominados pelo fornecedor .

** B = Baixa intensidade tecnológica; MB = Média Baixa intensidade tecnológica; MA = Média Alta intensidade tecnológica; A = Alta intensidade tecnológica. 
Quatro são os clusters formados a partir da análise de cluster realizada. O primeiro cluster formado (cluster I, figura 1) é composto por apenas uma atividade econômica da indústria de transformação, a atividade de fabricação de produtos de refino de petróleo (ID 12 - Refino, figura 1). Esse agrupamento representa 3,57\% das atividades selecionadas. $\mathrm{O}$ isolamento dessa atividade econômica no cluster deve ser justificado principalmente pelo distanciamento dos indicadores dessa atividade frente às outras atividades econômicas da indústria de transformação brasileira. Conforme tabela 2, o cluster I tem características bastante peculiares quando comparada as demais atividades econômicas em análise.

Como é possível observar na tabela 2, a exceção de máquinas (Maq) e softwares (Soft), os indicadores desse cluster apresentam valores superiores aos apresentados pela indústria de transformação como todo. A exemplo disso, tem-se o indicador de taxa de inovação tecnológica (TaxInov) que é de $52,36 \%$ para a atividade de refino de petróleo e de 36,3\% da atividade da indústria de transformação brasileira.

Já comparado aos indicadores dos outros clusters (II, III e IV), o cluster I exibe o segundo maior indicador de taxa de inovação (TaxInov: 52,36\%) e P\&D (PD: 0,9). Além disso, apesenta os menores valores em aquisição de máquina (Maq: 0.01\%) e software (Solf: 0,11\%), o que indica que desse cluster deriva maiores esforços de produção de conhecimento interno via P\&D. Assim, pode-se afirmar que o cluster I, formado pela atividade de refino de petróleo, possui uma alta-média atividade inovativa. Portanto, os resultados dos indicadores deste cluster são bastante divergentes da classificação da OCDE (2011) no qual pressupõe que essa atividade apresenta média-baixa intensidade tecnológica.

Ademais, o cluster I apresenta valores superiores dos indicadores de escolaridade (Escol), empregados em ciência e tecnologia $(\boldsymbol{C} \boldsymbol{T})$ e produtividade ( $\boldsymbol{R} \boldsymbol{V} \boldsymbol{V}$ e $\boldsymbol{V T \boldsymbol { T }})$, tabela 2. Como exemplo, o cluster I exibe 66,3\% de empregados com ensino superior (Escol) enquanto no cluster IV é de apenas 21,3\% ainda que seja o segundo cluster com melhor indicador. Da mesma maneira o cluster I apresenta em média 18,5\% de pesquisadores e engenheiros empregados $(\boldsymbol{C T})$, indicador este que está bem acima dos outros clusters que é de apenas $0,3 \% 1,5 \%$ e 2,8\%, respectivamente para os clusters II, III e IV.

Esse resultado é importante para demonstrar as peculiaridades de algumas atividades da indústria brasileira, como a atividade de refino de petróleo. Além do que reforça e estende os achados de Silva e Suzigan (2014) por demonstrarem que o setor de "Coque, petróleo e biocombustíveis" que contém a atividade de refino é o outlier que em termos de fontes de inovação, principalmente via P\&D interna. Assim, a atividade de refino de petróleo no Brasil apresenta uma dinâmica própria e diferenciada com índices de atividades da alta intensidade tecnológica e qualificação da mão e obra, divergindo do que é proposto pela classificação da OCDE. Esse resultado pode ser justificado dentre outros, pela crescente inserção de profissionais com maior nível de escolaridade que ocorreu ao longo das últimas décadas no Brasil e o elevado esforço de desenvolvimento tecnológico articulado com um sistema de instituições formais e informais conforme salienta Maciente e Araújo (2011).

Destaque também deve ser dado aos indicadores de produtividade do cluster I. Isso porque conforme é possível observar na tabela 2 a receita líquida de vendas $(\boldsymbol{R} \boldsymbol{L} \boldsymbol{V})$ do cluster I, formado apenas pela atividade de refino de petróleo, é de 4.367,8 e o valor da transformação industrial (VTI) é de 2.089,4, valores bem superiores aos encontrados nos outros clusters $(I I, I I I, I V)$.

Assim, o resultado do cluster I apresenta divergência a classificação da OCDE, entretanto, demonstra aderência à taxonomia de Pavitt (1984), uma vez que essa atividade é classificada no grupo das atividades de Intensiva em Escala que pressupõe considerável esforço de inovação via inovação de processo e grandes empresas, como apresentado nos indicadores nessa análise.

Já o cluster II é formado por $35,71 \%$ das atividades econômicas selecionadas, figura 1, são elas: fabricação de produtos têxteis (ID 4, têxtil); confecção de artigos do vestuário e acessórios (ID 5, vestuário); preparação de couros e fabricação de artefatos de couro, artigos de viagem e calçados (ID 6, couro); fabricação de produtos de madeira (ID 7, madeira); impressão e reprodução de gravações (ID 10, impressão); fabricação de coque e biocombustíveis (álcool e outros) (ID 11, biocombustíveis); fabricação de produtos de minerais não metálicos (ID 16, mineral); fabricação de produtos de metal, exceto máquinas e equipamentos (ID 18, metal); fabricação de móveis (ID 25, móveis); e fabricação de produtos diversos (ID 26, diversos). 
Ao avaliar os indicadores nesse cluster (II) é possível apontar que, a exceção de máquinas (Maq) e softwares (Soft), o cluster II exibe os menores valores médios dos indicadores de inovação, mão de obra e produtividade. Ademais, os mais altos valores dos indicadores de aquisição de máquinas (Maq: 0,11\%) e software (Soft: 1,56\%). Assim, os resultados sugerem que o cluster II apresenta baixos esforços tecnológicos internos e elevado acesso ao conhecimento via incorporação de inovações externas e concentradas em processos. Portanto, revela uma elevada aderência à classificação da OCDE e a taxonomia de Pavitt (1984) por demonstrar que esse cluster (II) possui baixa e média-baixa intensidade tecnológica, ou ainda, é um conjunto de atividade dominado pelos fornecedores que pressupõe reduzido esforço inovador e firmas de pequeno porte.

Entretanto, ao avaliar separadamente atividades que compõe o cluster II pode-se afirmar que as atividades de fabricação de coque e biocombustíveis (álcool e outros) (ID 11); fabricação de produtos de minerais não metálicos (ID 16); fabricação de produtos de metal, exceto máquinas e equipamentos (ID 18), demostram divergência com a taxonomia de Pavitt (1984), visto que de acordo com Pavitt essas atividades são classificadas de Produção Intensiva em Escala que pressupõe uma proeminência em inovação de processo e esforços inovador, resultado diferente do encontrado nessa análise.

O cluster III, por sua vez, é formado por $46,43 \%$ das atividades selecionadas nessa análise, figura 1, são elas: fabricação de produtos alimentícios (ID 1, alimento); fabricação de bebidas (ID 2, bebida); fabricação de produtos do fumo (ID 3, fumo); fabricação de celulose e outras pastas (ID 8; pasta), fabricação de papel, embalagens e artefatos de papel (ID 9, papel); fabricação de produtos químicos (ID 13, químico); fabricação de artigos de borracha e plástico (ID 15, borracha); metalurgia (ID 17, metalurgia), fabricação de máquinas, aparelhos e materiais elétricos (ID 21, material elétrico); fabricação de máquinas e equipamentos (ID 22, máquinas e equipamentos); fabricação de veículos automotores, reboques e carrocerias (ID 23, veículo); fabricação de instrumentos e mat. para uso médico e odontológico e de artigos ópticos (ID 27, odontológico) e manutenção, reparação e instalação de máquinas e equipamentos (ID 28, manutenção).

Ao avaliar os indicadores do cluster III é possível apontar que a dimensão da atividade inovativa configura-se com magnitude idêntica da indústria de transformação nacional (TaxInov: 36,30 e PD:0,7), e os indicadores de qualificação da mão de obra e produtividade um pouco superior à média nacional.

Ao comparar os indicadores do cluster III aos dos outros clusters (I, II e IV) é possível perceber que em termos de produtividade o cluster III (VTI: 209,9 e $\boldsymbol{R} \boldsymbol{L} \boldsymbol{V}: 465,0)$ é menor apenas do cluster I (VTI: 2089,4 e $\boldsymbol{R} \boldsymbol{L} \boldsymbol{V}:$ 4367,8). Outro exemplo, enquanto que em relação aos indicadores das dimensões de atividade inovativa e qualificação da mão de obra apresenta superioridade apenas comparada ao cluster II, o cluster III exibe 13,8\% empregados com ensino superior (Escol) enquanto a média da indústria de transformação é de 9,5\%. Assim, esse cluster (III) apresenta média-baixa atividade inovativa e qualificação da mão de obra e média-alta produtividade.

Em relação as atividades econômicas individuais que compõe o cluster III, é possível notar elevada heterogeneidade interna entre elas quando os indicadores são avaliados individualmente. A exemplo disso, as atividades de fabricação de produtos químicos (ID 13), fabricação de máquinas e equipamentos elétricos (ID 21), fabricação de instrumentos e materiais para uso médico e odontológico e de artigos ópticos (ID 27) apresentam taxa de inovação acima de $47 \%$. Por outro lado, é possível encontrar atividades como manutenção, reparação e instalação de máquinas e equipamentos (ID 28) que apresenta uma taxa de inovação (TaxInov) de apenas 22,92\%.

O mesmo ocorre em relação aos indicadores de produtividade em que a atividade de fabricação de celulose e outras pastas (ID 8) apresenta uma $\boldsymbol{R} \boldsymbol{L} \boldsymbol{V}$ de 964,5 enquanto que as atividades de fabricação de instrumentos e materiais para uso médico e odontológico e de artigos ópticos (ID 27) e manutenção, reparação e instalação de máquinas e equipamentos (ID 28) apresentam RVL de apenas 170,6 e 156,1, respectivamente. Entretanto, deve-se ressaltar que a análise realizada nesse trabalho ocorre a partir de um conjunto de diferentes indicadores de distintas dimensões conjuntamente de modo que atividade que apresentam disparidade em termos de algum indicador é posto no mesmo cluster devido a outro indicador. Assim o cluster III demonstra a importância de se utilizar diferentes dimensões para se avaliar a aderência das taxonomias frente a indústria nacional em detrimento de apenas um ou vários separadamente. 
Por fim, o cluster IV é formado pelas atividades: Fabricação de produtos farmoquímicos e farmacêuticos (ID 14, farmacêutica), Fabricação de equipamentos de informática, produtos eletrônicos e ópticos. Exceto ID 20 (ID 19, eletrônico), Fabricação de equipamentos de informática e periféricos (ID 20, informática) e Fabricação de outros equipamentos de transporte, exceto veículos automotores e Aviões (ID 24 , transporte), e equivale a $14,29 \%$ das atividades econômicas selecionadas.

No geral, o cluster IV apresentam desempenho superior à média da indústria de transformação nacional com os mais altos indicadores de taxa de inovação (TaxInov), P\&D (PD) e de empregados nas ocupações técnicas $(\boldsymbol{T e c})$. Além disso, percebe-se elevada aderência à taxonomia Pavitt (1984) e OCDE (2011) sob as três dimensões propostas neste trabalho, embora seja possível destacar particularidades dos resultados em determinada atividade econômica.

Ao analisar a primeira dimensão é importante ressaltar o baixo indicador de $P D(1,1)$ da atividade de fabricação de equipamentos de informática e periféricos (ID 20), número este que é inferior ao do agrupamento IV que é de 1,9 , embora seja superior à média nacional $(P D=0,7)$. Este comportamento pode ser explicado pelo predomínio de empresas transnacionais nesses setores, com baixo conteúdo local e a localização das atividades tecnológicas nas suas respectivas nações sede.

Quanto à segunda dimensão, o indicador $\boldsymbol{C T}$ da atividade de fabricação de produtos farmoquímicos e farmacêuticos (ID 14) e fabricação de equipamentos de informática e periféricos (ID 20) está abaixo do seu agrupamento (IV) (ID 14: 1,2\%; ID 20: 2,0\%; CT cluster IV: 2,8\%). Tal comportamento, justifica-se pela ampla fragmentação produtiva ocorrida nestes setores no contexto internacional. Segundo Vargas (2009) as atividades de maior intensidade tecnológica associadas ao processo de P\&D e à produção de princípios ativos da indústria de farmoquímicos e farmacêutica tendem a concentrar em países desenvolvidos, ficando para as filiais alocadas em países menos desenvolvidos, como o caso do Brasil, com a produção (formulação) de medicamentos e aplicação de testes clínicos com metodologias desenvolvidas externamente.

Por fim, a produtividade setorial em termos médios tanto o indicador $\boldsymbol{R} \boldsymbol{L} \boldsymbol{V}(463,2)$ como $\boldsymbol{V T I}(166,7)$ são superiores à média nacional ( $\boldsymbol{R} \boldsymbol{V} \boldsymbol{V}: 340,3$ e $\boldsymbol{V T I}: 137,7)$, embora esteja colocado na terceira posição quando comparado ao demais clusters, indicadores superiores apenas do cluster II (caracterizado por atividades de baixa produtividade). Esse resultado comporta-se divergente ao esperado, uma vez que quanto maior a intensidade e padrão tecnológico maiores seriam o indicador de produtividade setorial.

Em vista do exposto e com base na estrutura industrial, os resultados reforçam as conjeturas de que as atividades intensivas em escala no Brasil alocam proporcionalmente um maior número de empregados com caraterísticas ocupacionais vinculadas à $C, T \& I$. Além disso, são nestes setores, especialmente, no refino de petróleo que encontram os mais elevados indicadores de esforços inovativos e tecnológicos no país. Em termos gerais, os agrupamentos apresentam relativa aderência as categoriais setoriais propostas pela taxonomia Pavitt (1984) e classificação OCDE (2011). Contudo, há peculiaridade das atividades econômicas na indústria de transformação brasileira. Essa peculiaridade pode ser justificada em parte pela heterogeneidade inter e intrasetorial que é uma característica marcante da indústria de transformação de países em desenvolvimento. Assim, diferentemente dos países desenvolvidos no qual possuem uma estrutura produtiva diversificada e homogênea os países em desenvolvimento tendem a apresentarem um sistema de produção mais especializado e bastante heterogêneo, o que pode levar o progresso técnico interno a se difundir de forma lenta e desigual (Vasconcelos e Nogueira (2011), Catela e Porcile (2013)). Neste contexto, observa-se um grande desafio a ciência em adequar aos padrões propostos pelas taxonomias, uma vez que as taxonomias industriais são fundamentalmente rígidas e refletem uma ideologia e estrutura organizacional dos países desenvolvidos, e que a dessemelhança é intrínseca a dada estrutura produtiva e especificidades entre setores, segmentos, firmas e regiões, especialmente, no Brasil.

\section{Considerações finais}

O presente trabalho teve como objetivo analisar a aderência dos padrões setoriais das atividades econômicas da indústria de transformação brasileira com as duas classificações tecnológicas mais 
amplamente empregadas por formuladores de política e por pesquisadores da área de economia da inovação - Taxonomia Pavitt (1984) e classificação por intensidade tecnológica OCDE (2011) -.

Para tal, foi desenvolvido o problema de pesquisa que buscou investigar a existência da aderência das taxonomias industriais amplamente empregadas à estrutura produtiva das economias em desenvolvimento, e particularmente, ao caso brasileiro.

Para responder a este questionamento o trabalho foi desenvolvido com base em dois eixos: teórico e metodológico/empírico.

Do ponto de vista teórico, o trabalho está respaldado pelo arcabouço teórico neoschumpeteriano, onde se buscou revisar e discutir a construção das taxonomias industriais, a partir das características conceituais, metodológicas e operacionais. Do ponto de vista metodológico/empírico empregou a técnica multivariada de análise de cluster por meio do método hierárquico aglomerativo.

Quanto aos resultados foi possível validar relativa aderência dos padrões setoriais da indústria de transformação brasileira à taxonomia Pavitt (1984) e OCDE (2011), especialmente, setores categorizados como dominado pelo fornecedor e baixa intensidade tecnológica (concentrados no cluster II), em que exibem baixos indicadores de esforços tecnológicos e dependência de conhecimento externo representado pela elevada participação média dos indicadores Soft e Maq.

Foi possível identificar um expressivo esforço tecnológico nos setores industriais categorizados com produção intensiva em escala, especialmente, na atividade de refino de petróleo ID 12 (cluster I) que converge com a taxonomia Pavitt (1984) e difere da categoria proposta pela OCDE (2011), onde setores de média baixa intensidade tecnologia apresentam baixas oportunidades tecnológicas.

Concomitantemente, setores baseados em ciência e classificados com alta intensidade tecnológica segundo taxonomia Pavitt (1984) e classificação OCDE (2011), exibiram indicadores inferiores ao esperado para o padrão setorial a que pertencem, e razoáveis quando comparados as demais atividades econômicas (diga-se: setores de instrumentos, materiais para uso médico, odontológico e de artigos ópticos ID 20, Cluster III). Tal comportamento justifica-se pela elevada participação e atuação de firmas com capital estrangeiro no país, onde o desenvolvimento de atividades com maior intensidade tecnológica associadas ao processo de $\mathrm{P} \& \mathrm{D}$ concentram nos países sede ficando as filiais com atividade com menor incorporação de conteúdo tecnológico.

Contudo tal inflexão não invalida a utilização da taxonomia Pavitt (1984), e a classificação por intensidade tecnológica OCDE (2011) em relação à indústria nacional, mas faz necessária a identificação e análise das diferenças das estruturas produtivas. Logo, entender os padrões inovativos das economias distantes da fronteira tecnológica internacional são de grande relevância, pois os setores industriais são distintos do ponto de vista das complexidades industriais e intensidades tecnológicas. Portando, faz-se necessário a construção de indicadores que reproduzam novos olhares e que indiquem ao longo do processo possibilidades de mensuração dos padrões inovativos, tendo em vista, que as taxonomias industriais são instrumentos de observação para um conjunto de fenômenos complexos da economia, e apoiam o direcionamento de políticas setoriais e tomada de decisões para o uso de recursos públicos.

\section{Referências}

AREND, M. 50 Anos de Industrialização do Brasil (1955-2005): uma análise evolucionária. 2009. 252f. Tese de Doutorado em Economia, Programa de Pós Graduação em Economia, Universidade Federal do Rio Grande do Sul, Porto Alegre, 2009.

CAMPOS, B.; RUIZ, A. U. Padrões setoriais de inovação na indústria brasileira. Revista Brasileira de Inovação, v. 8, n. 1 jan/jun, 2009. p. 167-210.

CARLIN, D. O.; CHEVARRIA, D. G. Investigação da Relação Entre a Estrutura de Capital e Inovação e Intensidade Tecnológica nas Companhias Abertas Brasileiras: Uma análise com base na Taxonomia de Pavitt. In: XXVI SIMPÓSIO DE GESTÃO DA INOVAÇÃO TECNOLÓGICA, 26, 2012, Salvador. Simpósio. Salvador: Anpad, 2012. p.1-15. Disponível em: <http://www.anpad.org.br/diversos/trabalhos/Simpósio/simposio_2012/2012_SIMPOSIO143 pdf>. Acesso em: 02 maio 2018. 
CARVALHO, A. et al. Clusterização hierárquica espacial com atributos binários. Texto para Discussão, Instituto de Pesquisa Econômica Aplicada (IPEA), 2009.

CASTRO, D. F. Padrões setoriais da inovação tecnológica na indústria brasileira: uma análise de cluster a partir da PINTEC. 2010. 125 f. Dissertação (Mestrado em Economia Política). Pontifica Universidade Católica de São Paulo, São Paulo, 2010.

CATELA, E. S. E PORCILE, G. HETEROGENEIDADE ESTRUTURAL NA PRODUTIVIDADE DAS FIRMAS BRASILEIRAS. BRASÍLIA, DF: CEPAL. ESCRITÓRIO NO BRASIL/IPEA, 2013 (TEXTOS PARA DISCUSSÃO CEPAL-IPEA, 55).

CAVALCANTE, L. R. Classificações tecnológicas: uma sistematização. Nota Técnica, n. 17. Instituto de Pesquisa Econômica Aplicada (IPEA), Brasília, 2014.

DOSI, G. Technological paradigms and technological trajectories: a suggested interpretation of the determinants and directions of technical change. Research policy, v. 11, n. 3. p. 147-162, 1982.

DOSI, G. et al. The nature of the innovative process. Technical change and economic theory, v. 2. 1988. p. 590-607.

FEIJÓ, C. A.; CARVALHO, P; ALMEIDA, J. S. G. Ocorreu uma desindustrialização no Brasil? São Paulo: IEDI, 2005.

FIELDING, A. Cluster and classification techniques for the biosciences. Cambridge: Cambridge University Press, 2007.

FURTADO, A. T.; CARVALHO, R. Q. PADRÕES DE INTENSIDADE TECNOLÓGICA DA INDÚSTRIA BRASILEIRA: Um estudo comparativo com os países centrais. São Paulo em Perspectiva, São Paulo, v. 19, n. 1, p.70-84, mar. 2005.

GONÇALVES, E.; SIMÕES, R. Padrões de esforço tecnológico da indústria brasileira: uma análise setorial a partir de técnicas multivariadas. Revista Economia, 2005.

GUIDOLIN, S.M; MARTINELLI, O. Regimes tecnológicos da indústria brasileira: uma contribuição para a análise empírica. In: Encontro Nacional dos Centros Nacionais de Pós-Graduação em Economia ANPEC, 36, Salvador, Dez. 9-12, 2008.

HAIR, J. et al. Análise multivariada de dados. Bookman Editora, 2009.

HATZICHRONOGLOU, T. Revision of the high-technology sector and product classification. OECD Science, Technology and Industry Working Papers, 1997 (working paper n 1997/02).

INSTITUTO BRASILEIRO DE GEOGRAFIA E ESTATÍSTICA (IBGE). Coordenação de Indústria. Pesquisa de inovação 2014. Rio de Janeiro, 2016a.

. (IBGE). Pesquisa Industrial - Empresa 2014. Rio de Janeiro, 2016 b.

(IBGE). Classificações Estatísticas e a comissão nacional de classificação. Disponível em:

$<$ http://cnae.ibge.gov.br/busca-online-

cnae. $h$ tml divisao $=18 \&$ tipo $=$ cnae $\&$ versao $=6 \&$ view $=$ divisao $>$. Acesso em: 02 de fev de 2018.

JOHNSON, S. C. Hierarchical clustering schemes. Psychometrika, v. 32, n. 3, p. 241-254, 1967.

LINDEN, R. Técnicas de agrupamento. Revista de Sistemas de Informação da FSMA, v. 1, n. 4, p. 1836, 2009.

MACIENTE, A. N.; ARAÚJO, T. C. A demanda por engenheiros e profissionais afins no mercado de trabalho formal. Radar, Brasília: Ipea, n. 12, fev. 2011.

MORCEIRO, P. C. et al. Por que não baixa tecnologia. In: Proceedings of the 9th GLOBELICS International Conference. 2011.

NELSON, R.; WINTER, S. Evolutionary Theory of Economic Change. Cambridge MA. Belknap Press of Harvard University Press, 1982.

ORGANIZAÇÃO PARA A COOPERAÇÃO E DESENVOLVIMENTO ECONÔMICO - OCDE. Science, Technology and Industry Scoreboard. Paris: OCDE, 2011. ISIC REV. 3 TECHNOLOGY INTENSITY DEFINITION. Paris: OCDE, 2011.

PAVITT, K. Sectoral patterns of technical change: towards a taxonomy and a theory. Research Policy, v.13, p. 343-373, 1984.

RAIS. Relação Anual de Informações Sociais. Ministério do Trabalho e Emprego (MTE). Disponível em: http://www.rais.gov.br/. Acesso em: 15 de nov. de 2017. 
ROBSON, M.; TOWNSEND, J.; PAVITT, K. Sectoral patterns of production and use of innovations in the UK. Research Policy, 17: p.1-14, 1988.

ROHLF, F. J. Adaptative hierarquical clustering schemes. Systematic Zoology, v.19, n.1, p.58-82, 1970.

SBRAGIA, R.; KRUGLIANSKAS, I.; ARANGO-ALZATE, T. Empresas inovadoras no Brasil: uma proposição de tipologia e características associadas, FEA/USP: Série Working Papers, n.001/003, 2002. Disponível em http://www.ead.fea.usp.br/wpapers. Acesso em 10/05/2018.

SILVA, E. H. Sectoral taxonomy with indicators of innovative effort. Revista de Economia Contemporânea, v. 17, n. 1, p. 129-152, 2013.

SILVA, C. F; SUZIGAN, W. Padrões setoriais de inovação da indústria de transformação brasileira. Estudos Econômicos (São Paulo), v. 44, n. 2, p. 277-321, 2014.

VARGAS, M. A et al. Reestruturação na indústria farmacêutica mundial e seus impactos na dinâmica produtiva e inovativas do setor farmacêutico brasileiro. Artigo apresentado a sessões ordinárias do GIS (Grupo de pesquisa e "Complexo Industrial e inovação em Saúde”, da Fundação Oswaldo Cruz), 2009.

VASCONCELOS, Lucas Ferraz; NOGUEIRA, Mauro Oddo. Heterogeneidade estrutural no setor industrial. Radar: tecnologia, produção e comércio exterior, n. 14, p. 21-26. Brasília: Ipea, jun. 2011. Disponível em: http://www.ipea.gov.br/portal/images/stories/PDFs/radar/110727_radar14.pdf

YONAMINI, F. M. Nova taxonomia de regimes tecnológicos para o caso de um país em desenvolvimento como o Brasil. 2011. 109 f, Tese de Doutorado, Curso de Programa de Pósgraduação em Desenvolvimento Econômico, Universidade Federal do Paraná, Curitiba, 2011. 
Anexo A - Nível de agregação e descrição setorial da base de dados segundo CNAE 2.0

\begin{tabular}{|c|c|c|c|c|c|}
\hline \multirow[b]{2}{*}{ Atividade econômica } & \multirow{2}{*}{\multicolumn{2}{|c|}{ Sigla }} & \multirow[b]{2}{*}{$\begin{array}{l}\text { Classificação } \\
\text { CNAE 2.0 }\end{array}$} & \multicolumn{2}{|l|}{ Taxonomia } \\
\hline & & & & $\begin{array}{c}\text { Pavitt (1984) } \\
\text { (Categoria setorial) }\end{array}$ & $\begin{array}{l}\text { OCDE (2011) } \\
\text { (Intensidade } \\
\text { tecnológica) }\end{array}$ \\
\hline Fabricação de produtos alimentícios & $(1)$ & Alimento & Divisão 10 & Produção intensiva em escala & Baixa \\
\hline Fabricação de bebidas & $(2)$ & Bebida & Divisão 11 & Produção intensiva em escala & Baixa \\
\hline Fabricação de produtos do fumo & $(3)$ & Fumo & Divisão 12 & Produção intensiva em escala & Baixa \\
\hline Fabricação de produtos têxteis & (4) & Têxtil & Divisão 13 & Dominado pelo fornecedor & Baixa \\
\hline Confecção de artigos do vestuário e acessórios & $(5)$ & Vestuário & Divisão 14 & Dominado pelo fornecedor & Baixa \\
\hline Preparação de couros e fabricação de artefatos de couro, artigos para viagem e calçados & (6) & Couro & Divisão 15 & Dominado pelo fornecedor & Baixa \\
\hline Fabricação de produtos de madeira & $(7)$ & Madeira & Divisão 16 & Dominado pelo fornecedor & Baixa \\
\hline Fabricação de celulose e outras pastas para a fabricação de papel & $(8)$ & Pasta & Grupo 17.1 & Dominado pelo fornecedor & Baixa \\
\hline Fabricação de celulose, papel e produtos de papel. Exceto Grupo 17.1 & $(9)$ & Papel & Divisão 17 & Dominado pelo fornecedor & Baixa \\
\hline Impressão e reprodução de gravações & $(10)$ & Impressão & Divisão 18 & Dominado pelo fornecedor & Baixa \\
\hline Fabricação de coque, de produtos derivados do petróleo e de biocombustíveis. Exceto Grupo 19.2 & (11) & Biocombustíveis & Divisão 19 & Produção intensiva em escala & Média-Baixa \\
\hline Refino de petróleo & (12) & Refino & Grupo 192 & Produção intensiva em escala & Média-Baixa \\
\hline Fabricação de produtos químicos & (13) & Químico & Divisão 20 & Baseado em Ciência & Média-Alta \\
\hline Fabricação de produtos farmoquímicos e farmacêuticos & (14) & Farmacêutico & Divisão 21 & Baseado em Ciência & Alta \\
\hline Fabricação de produtos de borracha e de material plástico & $(15)$ & Borracha & Divisão 22 & Produção intensiva em escala & Média-Baixa \\
\hline Fabricação de produtos de minerais não metálicos & $(16)$ & Mineral & Divisão 23 & Produção intensiva em escala & Média-Baixa \\
\hline Metalurgia & $(17)$ & Metalurgia & Divisão 24 & Produção intensiva em escala & Média-Baixa \\
\hline Fabricação de produtos de metal, exceto máquinas e equipamentos & (18) & Metal & Divisão 25 & Produção intensiva em escala & Média-Baixa \\
\hline Fabricação de equipamentos de informática, produtos eletrônicos e ópticos. Exceto Grupo 26.2 & $(19)$ & Eletrônico & Divisão 26 & Baseado em Ciência & Alta \\
\hline Fabricação de equipamentos de informática e periféricos & (20) & Informática & Grupo 26.2 & Baseado em Ciência & Alta \\
\hline Fabricação de máquinas, aparelhos e materiais elétricos & $(21)$ & Mat. Elétrico & Divisão 27 & Fornecedores especializados & Média-Alta \\
\hline Fabricação de máquinas e equipamentos & $(22)$ & $\begin{array}{l}\text { Máquinas e } \\
\text { equipamentos }\end{array}$ & Divisão 28 & Produção intensiva em escala & Média-Alta \\
\hline Fabricação de veículos automotores, reboques e carrocerias & $(23)$ & Veiculo & Divisão 29 & Produção intensiva em escala & Média-Alta \\
\hline Fabricação de outros equipamentos de transporte, exceto veículos automotores e Aviões & $(24)$ & Transporte & Divisão 30 & Produção intensiva em escala & Média-Alta \\
\hline Fabricação de móveis & $(25)$ & Móveis & Divisão 31 & Dominado pelo fornecedor & Baixa \\
\hline Fabricação de produtos diversos. Exceto Grupo 32.5 & $(26)$ & Diversos & Divisão 32 & Fornecedores especializados & Baixa \\
\hline Fabricação de instrumentos e materiais para uso médico e odontológico e de artigos ópticos & $(27)$ & Odontológico & Grupo 32.5 & Baseado em Ciência & Alta \\
\hline Manutenção, reparação e instalação de máquinas e equipamentos & $(28)$ & Manutenção & Divisão 33 & Fornecedores especializados & Média-Alta \\
\hline Indústria de transformação & & $\mathrm{C}$ & Seção C & - & - \\
\hline
\end{tabular}

Fonte: Elaboração própria (2018) baseado em Pavitt (1984), Hatzichronoglou (1997), Robinson et al (2003), OCDE (2011), Squeff (2012), Cavalcante

(2014) e (IBGE (2017). 
ANEXO B - Descrição dos grupos ocupacionais do pessoal ocupacional técnico científico selecionado conforme indicador $C T$ e Tec

\begin{tabular}{|c|c|c|}
\hline Indicador & Grupo ocupacional & (Código) Família \\
\hline \multirow[b]{2}{*}{$C T$} & Pesquisadores & $\begin{array}{l}\text { (2030) Pesquisadores das Ciências Biológicas; (2031) Pesquisadores das Ciências Naturais e Exatas; (2032) Pesquisadores de Engenharia } \\
\text { e Tecnologia; (2033) Pesquisadores das Ciências da Saúde; (2034) Pesquisadores das Ciências da Agricultura; (2035) Pesquisadores das } \\
\text { Ciências Sociais e Humanas. }\end{array}$ \\
\hline & Engenharias & $\begin{array}{l}\text { (2021) Engenheiros de Controle e Automação, Engenheiros Mecatrônicos e afins; (2140) Engenheiros Ambientais e afins; (2142) } \\
\text { Engenheiros Civis e afins; (2143) Engenheiros Eletricistas, Eletrônicos e afins; (2144) Engenheiros Mecânicos e afins; (2145) Engenheiros } \\
\text { Químicos e afins; (2146) Engenheiros Metalurgistas, de Materiais e afins; (2147) Engenheiros de Minas e afins; (2148) Engenheiros } \\
\text { Agrimensores e Engenheiros Cartógrafos; (2149) Engenheiros de Produção, Qualidade, Segurança e afins; (2122) Engenheiros em } \\
\text { Computação; (2221) Engenheiros Agrossilvipecuários; (2222) Engenheiros de Alimentos e afins. }\end{array}$ \\
\hline Tec & Técnicos & $\begin{array}{l}\text { (3001) Técnicos em Mecatrônica; (3003) Técnicos em Eletromecânica; (3011) Técnicos de Laboratório Industrial; (3012) Técnicos de } \\
\text { apoio à Bioengenharia; (3111) Técnicos Químicos; (3112) Técnicos de Produção de Indústrias Químicas, Petroquímicas, Refino de } \\
\text { Petróleo, Gás e afins; (3113) Técnicos em Materiais, Produtos Cerâmicos e Vidros; (3114) Técnicos em Fabricação de Produtos Plásticos } \\
\text { e de Borracha; (3115) Técnicos em Controle Ambiental, Utilidades e Tratamento de Eluentes; (3116) Técnicos Tếxteis; (3131) Técnicos } \\
\text { em Eletricidade e Eletrotécnica; (3132) Técnicos em Eletrônica; (3133) Técnicos em Telecomunicações; (3134) Técnicos em Calibrações } \\
\text { e Instrumentação; (3141) Técnicos Mecânicos na Fabricação e Montagem de Máquinas, Sistemas e Instrumentos; (3142) Técnicos } \\
\text { Mecânicos (Ferramentas); (3143) Técnicos em Mecânica Veicular; (3144) Técnicos Mecânicos na Manutenção de Máquinas, Sistemas e } \\
\text { Instrumentos; (3146) Técnicos em Metalurgia (Estruturas Metálicas); (3147) Técnicos em Siderurgia; (3161) Técnicos em Geologia; } \\
\text { (3163) Técnicos em Mineração; (3171) Técnicos de Desenvolvimento de Sistemas e Aplicações; (3201) Técnicos em Biologia; (3211) } \\
\text { Técnicos Agrícolas; (3212) Técnicos Florestais; (3251) Técnico em Farmácia e em Manipulação Farmacêutica; (3253) Técnicos de Apoio } \\
\text { à Biotecnologia; (3421) Especialistas em Logística de Transportes; (3911) Técnicos de Planejamento e Controle de Produção; (3912) } \\
\text { Técnicos de Controle da Produção; (3951) Técnicos de Apoio em Pesquisa e Desenvolvimento. }\end{array}$ \\
\hline
\end{tabular}

Fonte: Elaboração própria (2018) baseado na CBO (2002) 